\title{
Hadamard-type fractional functional differential equations and inclusions with retarded and advanced arguments
}

\author{
Ravi P Agarwal' ${ }^{1}$, Sotiris K Ntouyas ${ }^{2,3}$, Bashir Ahmad ${ }^{3 *}$ and Abdullah K Alzahrani ${ }^{3}$
}

\section{"Correspondence:}

bashirahmad_qau@yahoo.com ${ }^{3}$ Nonlinear Analysis and Applied Mathematics (NAAM)-Research Group, Department of Mathematics, Faculty of Science, King Abdulaziz University, P.O. Box 80203, Jeddah, 21589, Saudi Arabia

Full list of author information is available at the end of the article

\begin{abstract}
In this paper, we establish sufficient conditions for the existence and uniqueness of solutions for boundary value problems of Hadamard-type fractional functional differential equations and inclusions involving both retarded and advanced arguments. We make use of the standard tools of fixed point theory to obtain the main results.
\end{abstract}

MSC: 34A08; 34K05

Keywords: functional differential equation; fractional derivative; retarded argument; advanced argument; existence

\section{Introduction}

Differential equations of fractional order play a very important role in describing many real world phenomena. The tools of fractional calculus are effectively employed in improving mathematical modeling of several problems in physics, mechanics and other fields. The theory of differential equations of fractional order has recently received great attention and now constitutes a significant branch of mathematical analysis. For details and examples, we refer the reader to a series of papers and monographs; for instance, see [1-20].

Functional differential equations arise in a variety of areas of biological, physical, and engineering applications, see, for example, the books of Kolmanovskii and Myshkis [21] and Hale and Verduyn Lunel [22], and the references cited therein. Recently, in [23], the authors studied the existence and uniqueness of solutions for fractional order Hadamardtype functional and neutral functional differential equations.

In this paper, motivated by [23], we study boundary value problems of Hadamard-type fractional functional differential equations and inclusions involving both retarded and advanced arguments. As a first problem, we consider

$$
\begin{aligned}
& D^{\alpha} x(t)=f\left(t, x^{t}\right), \quad 1 \leq t \leq e, 1<\alpha<2, \\
& x(t)=\chi(t), \quad 1-r \leq t \leq 1 \\
& x(t)=\psi(t), \quad e \leq t \leq e+h,
\end{aligned}
$$

(c) 2016 Agarwal et al. This article is distributed under the terms of the Creative Commons Attribution 4.0 International License (http://creativecommons.org/licenses/by/4.0/), which permits unrestricted use, distribution, and reproduction in any medium, provided you give appropriate credit to the original author(s) and the source, provide a link to the Creative Commons license, and indicate if changes were made. 
where $D^{\alpha}$ is the Hadamard fractional derivative. Here, $f:[1, e] \times C([-r, h], \mathbb{R}) \rightarrow \mathbb{R}$ is a given function, $\chi \in C([1-r, 1], \mathbb{R})$ with $\chi(1)=0$ and $\psi \in C([e, e+h], \mathbb{R})$ with $\psi(e)=0$. For any function $x$ defined on $[1-r, e+h]$ and any $1 \leq t \leq e$, we denote by $x^{t}$ the element of $C([-r, h], \mathbb{R})$ defined by $x^{t}(\theta)=x(t+\theta)$ for $-r \leq \theta \leq h$, where $r, h \geq 0$ are constants.

In the second problem, we extend our study to the multi-valued case given by

$$
\begin{aligned}
& D^{\alpha} x(t) \in F\left(t, x^{t}\right), \quad 1 \leq t \leq e, 1<\alpha<2, \\
& x(t)=\chi(t), \quad 1-r \leq t \leq 1, \\
& x(t)=\psi(t), \quad e \leq t \leq e+h,
\end{aligned}
$$

where $F:[1, e] \times C([-r, h], \mathbb{R}) \rightarrow \mathcal{P}(\mathbb{R})$ is a multi-valued map $(\mathcal{P}(\mathbb{R})$ is the family of all nonempty subjects of $\mathbb{R})$.

The paper is organized as follows. In Section 2 we recall some preliminary concepts. We obtain existence and uniqueness results for the problem (1)-(3) in Section 3. These results are based on a nonlinear alternative of Leray-Schauder type and the Banach contraction mapping principle. In Section 4, we present the existence results for convex and nonconvex multi-valued maps involved in the problem (4)-(6) which, respectively, rely on the nonlinear alternative of Leray-Schauder type and a fixed point theorem for contractive multi-valued maps due to Covitz and Nadler.

\section{Auxiliary facts and results}

This section is devoted to some definitions and results which will be needed throughout this paper.

By $C:=C([-r, h], \mathbb{R})$ we denote the Banach space of all continuous functions from $[-r, h]$ into $\mathbb{R}$ equipped with the norm

$$
\|\chi\|_{[-r, h]}=\sup \{|\chi(\theta)|:-r \leq \theta \leq h\}
$$

and $C([1, e], \mathbb{R})$ is the Banach space endowed with the norm $\|x\|_{0}=\sup \{|x(t)|: 1 \leq t \leq e\}$. Also, let $E=C([1-r, e+h], \mathbb{R}), E_{1}=C([1-r, 1], \mathbb{R})$, and $E_{2}=C([e, e+h], \mathbb{R})$ be, respectively, endowed with the norms $\|x\|_{[1-r, e+h]}=\sup \{|x(t)|: 1-r \leq t \leq e+h\},\|x\|_{[1-r, 1]}=\sup \{|x(t)|$ : $1-r \leq t \leq 1\}$, and $\|x\|_{[e, e+h]}=\sup \{|x(t)|: e \leq t \leq e+h\}$.

Definition 2.1 [7] The Hadamard derivative of fractional order $q$ for a function $g$ : $[1, \infty) \rightarrow \mathbb{R}$ is defined as

$$
D^{q} g(t)=\frac{1}{\Gamma(n-q)}\left(t \frac{d}{d t}\right)^{n} \int_{1}^{t}\left(\log \frac{t}{s}\right)^{n-q-1} \frac{g(s)}{s} d s, \quad n-1<q<n, n=[q]+1,
$$

where $[q]$ denotes the integer part of the real number $q$ and $\log (\cdot)=\log _{e}(\cdot)$.

Definition 2.2 [7] The Hadamard fractional integral of order $q$ for a function $g$ is defined as

$$
I^{q} g(t)=\frac{1}{\Gamma(q)} \int_{1}^{t}\left(\log \frac{t}{s}\right)^{q-1} \frac{g(s)}{s} d s, \quad q>0,
$$

provided the integral exists. 
Lemma 2.3 Given $g \in A C([1, e], \mathbb{R})$ and $1<\alpha \leq 2$, the solution $u \in C^{2}([1, e], \mathbb{R})$ of the problem

$$
\begin{aligned}
& D^{\alpha} u(t)=g(t), \quad 0<t<1, \\
& u(1)=u(e)=0,
\end{aligned}
$$

is equivalent to the integral equation

$$
u(t)=-\int_{1}^{e} G(t, s) \frac{g(s)}{s} d s
$$

where

$$
G(t, s)=\frac{1}{\Gamma(\alpha)} \begin{cases}(\log t)^{\alpha-1}(1-\log s)^{\alpha-1}-(\log t-\log s)^{\alpha-1}, & 1 \leq s \leq t \leq e \\ (\log t)^{\alpha-1}(1-\log s)^{\alpha-1}, & 1 \leq t \leq s \leq e\end{cases}
$$

Proof As argued in [7], the solution of the Hadamard differential equation (7) can be written as

$$
x(t)=\frac{1}{\Gamma(\alpha)} \int_{1}^{t}\left(\log \frac{t}{s}\right)^{\alpha-1} \frac{g(s)}{s} d s+c_{1}(\log t)^{\alpha-1}+c_{2}(\log t)^{\alpha-2} .
$$

Using the given boundary conditions, we find that $c_{2}=0$ and

$$
c_{1}=-\frac{1}{\Gamma(\alpha)} \int_{1}^{e}\left(\log \frac{e}{s}\right)^{\alpha-1} \frac{g(s)}{s} d s .
$$

Substituting the values of $c_{1}$ and $c_{2}$ in (11), we obtain

$$
\begin{aligned}
u(t)= & \frac{1}{\Gamma(\alpha)} \int_{1}^{t}\left(\log \frac{t}{s}\right)^{\alpha-1} \frac{g(s)}{s} d s-(\log t)^{\alpha-1} \frac{1}{\Gamma(\alpha)} \int_{1}^{e}\left(\log \frac{e}{s}\right)^{\alpha-1} \frac{g(s)}{s} d s \\
= & \frac{1}{\Gamma(\alpha)} \int_{1}^{t}\left(\log \frac{t}{s}\right)^{\alpha-1} \frac{g(s)}{s} d s-(\log t)^{\alpha-1} \frac{1}{\Gamma(\alpha)} \int_{1}^{t}\left(\log \frac{e}{s}\right)^{\alpha-1} \frac{g(s)}{s} d s \\
& -(\log t)^{\alpha-1} \frac{1}{\Gamma(\alpha)} \int_{t}^{e}\left(\log \frac{e}{s}\right)^{\alpha-1} \frac{g(s)}{s} d s \\
= & -\frac{1}{\Gamma(\alpha)} \int_{1}^{t}\left[(\log t)^{\alpha-1}(1-\log s)^{\alpha-1}-(\log t-\log s)^{\alpha-1}\right] \frac{g(s)}{s} d s \\
& -\int_{t}^{e} \frac{1}{\Gamma(\alpha)}(\log t)^{\alpha-1}(1-\log s)^{\alpha-1} \frac{g(s)}{s} d s \\
= & -\int_{1}^{e} G(t, s) \frac{g(s)}{s} d s,
\end{aligned}
$$

where $G(t, s)$ is given by $(10)$. The converse of the theorem follows by direct computation. This completes the proof.

Now we recall some facts from multi-valued analysis. Let $(X,\|\cdot\|)$ be a Banach space. Let $\mathcal{P}(X)=\{Y \subset X: Y \neq \emptyset\}, \mathcal{P}_{b}(X)=\{Y \in \mathcal{P}(X): Y$ bounded $\}, \mathcal{P}_{c l}(X)=\{Y \in$ 
$\mathcal{P}(X): Y$ closed $\}, \mathcal{P}_{b, c l}(X)=\{Y \in \mathcal{P}(X): Y$ bounded and closed $\}, \mathcal{P}_{c p}(X)=\{Y \in \mathcal{P}(X):$ $Y$ compact $\}, \mathcal{P}_{c p, c}(X)=\{Y \in \mathcal{P}(X): Y$ compact and convex $\}$.

A multi-valued map $G: X \rightarrow \mathcal{P}(X)$ has convex (closed) values if $G(x)$ is convex (closed) for all $x \in X$. We say that $G$ is bounded on bounded sets if $G(B)$ is bounded in $X$ for each bounded set $B$ of $X$, i.e., $\sup _{x \in B}\{\sup \{\|y\|: y \in G(x)\}\}<\infty$. The map $G$ is called upper semicontinuous (u.s.c.) on $X$ if for each $x_{0} \in X$ the set $G\left(x_{0}\right)$ is a nonempty, closed subset of $X$, and if for each open set $N$ of $X$ containing $G\left(x_{0}\right)$, there exists an open neighborhood $M$ of $x_{0}$ such that $G(M) \subseteq N$. Also, $G$ is said to be completely continuous if $G(B)$ is relatively compact for every bounded subset $B \subseteq X$. If the multi-valued map $G$ is completely continuous with nonempty compact values, then $G$ is u.s.c. if and only if $G$ has a closed graph (i.e., $x_{n} \rightarrow x_{*}, y_{n} \rightarrow y_{*}, y_{n} \in G\left(x_{n}\right)$ imply $\left.y_{*} \in G\left(x_{*}\right)\right)$. Finally, we say that $G$ has a fixed point if there exists $x \in X$ such that $x \in G(x)$.

A multi-valued map $G: J \rightarrow \mathcal{P}_{c l}(X)$ is said to be measurable if, for each $x \in E$, the function $Y: J \rightarrow X$ defined by

$$
Y(t)=\operatorname{dist}(x, G(t))=\inf \{\|x-z\|: z \in G(t)\}
$$

is Lebesgue measurable.

Definition 2.4 A multi-valued map $F: J \times C([-r, h], \mathbb{R}) \rightarrow \mathcal{P}_{c p, c}(\mathbb{R})$ is said to be $L^{1}$ Carathéodory if

(i) $t \mapsto F(t, x)$ is measurable, for each $x \in C([-r, h], \mathbb{R})$,

(ii) $x \mapsto F(t, x)$ is upper semicontinuous for almost all $t \in J$, and

(iii) for each real number $\rho>0$, there exists a function $h_{\rho} \in L^{1}\left(J, \mathbb{R}^{+}\right)$such that

$$
\|F(t, u)\|:=\sup \{|v|: v \in F(t, u)\} \leq h_{\rho}(t), \quad \text { a.e. } t \in J
$$

for all $u \in C([-r, h], \mathbb{R})$ with $\|u\| \leq \rho$.

Let $(X, d)$ be a metric space induced from the normed space $(X,\|\cdot\|)$. Consider $H_{d}$ : $\mathcal{P}(X) \times \mathcal{P}(X) \longrightarrow \mathbb{R}_{+} \cup\{\infty\}$, given by

$$
H_{d}(\mathcal{A}, \mathcal{B})=\max \left\{\sup _{a \in \mathcal{A}} d(a, \mathcal{B}), \sup _{b \in \mathcal{B}} d(\mathcal{A}, b)\right\},
$$

where $d(\mathcal{A}, b)=\inf _{a \in \mathcal{A}} d(a, b), d(a, \mathcal{B})=\inf _{b \in \mathcal{B}} d(a, b)$. Then $\left(\mathcal{P}_{b, c l}(X), H_{d}\right)$ is a metric space and $\left(\mathcal{P}_{c l}(X), H_{d}\right)$ is a generalized (complete) metric space.

Definition 2.5 A multi-valued operator $G: X \rightarrow \mathcal{P}_{c l}(X)$ is called

(a) $\gamma$-Lipschitz if there exists $\gamma>0$ such that

$$
H_{d}(G(x), G(y)) \leq \gamma d(x, y), \quad \text { for each } x, y \in X
$$

(b) a contraction if it is $\gamma$-Lipschitz with $\gamma<1$.

For more details on multi-valued maps we refer to the books of Deimling [24], Górniewicz [25], Hu and Papageorgiou [26], and Tolstonogov [27]. 


\section{Existence results for the problem (1)-(3)}

By a solution of (1)-(3) we mean a function $x \in C^{2}([1-r, e+h], \mathbb{R})$ that satisfies the equation $D^{\alpha} x(t)=f\left(t, x^{t}\right)$ on $[1, e]$ and the conditions $x(t)=\chi(t), \chi(1)=0$ on $[1-r, 1]$ and $x(t)=\psi(t)$, $\psi(e)=0$ on $[e, e+h]$.

Our existence result for the boundary value problem (1)-(3) is based on the following fixed point theorem.

Lemma 3.1 (Nonlinear alternative for single-valued maps ([28])) Let E be a Banach space, $C$ a closed, convex subset of $E, U$ an open subset of $C$ and $0 \in U$. Suppose that $F: \bar{U} \rightarrow C$ is a continuous, compact (that is, $F(\bar{U})$ is a relatively compact subset of $C$ ) map. Then either

(i) F has a fixed point in $\bar{U}$, or

(ii) there is a $u \in \partial U$ (the boundary of $U$ in $C$ ) and $\lambda \in(0,1)$ with $u=\lambda F(u)$.

Theorem 3.2 Let $f:[1, e] \times C([-r, h], \mathbb{R}) \rightarrow \mathbb{R}$ be a continuous function. Assume the following assumptions hold:

$\left(H_{1}\right)$ there exist $p \in C(J, \mathbb{R})$ and $\Omega:[0, \infty) \rightarrow(0, \infty)$ continuous and nondecreasing such that

$$
|f(t, u)| \leq p(t) \Omega\left(\|u\|_{[-r, h]}\right)
$$

for all $t \in J$ and all $u \in C([-r, h], \mathbb{R})$;

$\left(\mathrm{H}_{2}\right)$ there exists a number $K_{0}>0$ such that

$$
\frac{K_{0}}{\frac{2\|p\|_{0}}{\Gamma(\alpha+1)} \Omega\left(K_{0}+\max \left\{\|x\|_{[1-r, 1]},\|x\|_{[e, e+h]}\right\}\right)}>1
$$

Then the boundary value problem (1)-(3) has at least one solution on the interval $[1-r$, $e+h]$.

Proof To transform the problem (1)-(3) into a fixed point problem, we consider an operator $\mathcal{Q}: C([1-r, e+h], \mathbb{R}) \rightarrow C([1-r, e+h], \mathbb{R})$ defined by

$$
(\mathcal{Q} x)(t)= \begin{cases}\chi(t), & \text { if } t \in[1-r, 1], \\ \int_{1}^{e} G(t, s) \frac{f\left(s, x^{s}\right)}{s} d s, & \text { if } t \in[1, e], \\ \psi(t), & \text { if } t \in[e, e+h]\end{cases}
$$

Let $u:[1-r, e+h] \rightarrow \mathbb{R}$ be a function defined by

$$
u(t)= \begin{cases}\chi(t), & \text { if } t \in[1-r, 1] \\ 0, & \text { if } t \in[1, e] \\ \psi(t), & \text { if } t \in[e, e+h]\end{cases}
$$

For each $y \in C([1, e], \mathbb{R})$ with $y(1)=0$ we denote by $z$ the function defined by

$$
z(t)= \begin{cases}0, & \text { if } t \in[1-r, 1] \\ y(t), & \text { if } t \in[1, e] \\ 0, & \text { if } t \in[e, e+h]\end{cases}
$$


Let us set $x(t)=y(t)+u(t)$ such that $x^{t}=y^{t}+u^{t}$ for every $1 \leq t \leq e$, where

$$
\begin{aligned}
& x(t)=\int_{1}^{e} G(t, s) \frac{f\left(s, x^{s}\right)}{s} d s, \\
& y(t)=\int_{1}^{e} G(t, s) \frac{f\left(s, y^{s}+u^{s}\right)}{s} d s .
\end{aligned}
$$

Next, we define $B=\{y \in C([1-r, e+h], \mathbb{R}): y(1)=0\}$ and let $\mathfrak{S}: B \rightarrow B$ be an operator given by

$$
(\mathfrak{S} y)(t)= \begin{cases}0, & 1-r \leq t \leq 1 \\ \int_{1}^{e} G(t, s) \frac{f\left(s, y^{s}+u^{s}\right)}{s} d s, & 1 \leq t \leq e \\ 0, & e \leq t \leq e+h\end{cases}
$$

Then it is enough to show that the operator $\mathfrak{S}$ has a fixed point which will imply that the operator $\mathcal{Q}$ has a fixed point and in consequence, this fixed point will correspond to a solution of the problem (1)-(3). In the following three steps, it will be shown that the operator $\mathfrak{S}$ is continuous and completely continuous.

Step 1: $\mathfrak{S}$ is continuous.

Let $\left(y_{n}\right)$ be a sequence such that $y_{n} \rightarrow y$ in $B$. Then we have

$$
\begin{aligned}
\left|\left(\mathfrak{S} y_{n}\right)(t)-(\mathfrak{S} y)(t)\right| & \leq \int_{1}^{e} G(t, s)\left|f\left(s, y^{n s}+u^{s}\right)-f\left(s, y^{s}+u^{s}\right)\right| \frac{d s}{s} \\
& \leq\left\|f\left(\cdot, y^{n(\cdot)}+u^{(\cdot)}\right)-f\left(\cdot, y^{(\cdot)}+u^{(\cdot)}\right)\right\|_{0} \int_{1}^{e} G(t, s) \frac{d s}{s} .
\end{aligned}
$$

Since the function $f$ is continuous, we have

$$
\left\|\mathfrak{S} y_{n}-\mathfrak{S} y\right\|_{[1-r, e+h]} \leq\left\|f\left(\cdot, y^{n(\cdot)}+u^{(\cdot)}\right)-f\left(\cdot, y^{(\cdot)}+u^{(\cdot)}\right)\right\|_{0} \int_{1}^{e} G(t, s) \frac{d s}{s} \rightarrow 0 \quad \text { as } n \rightarrow \infty \text {. }
$$

Step 2: $\mathfrak{S}$ maps bounded sets into bounded sets in B.

For any $k>0$, it is enough to show that there exists a positive constant $\hat{L}$ such that, for each $y \in U_{k}:=\left\{y \in B:\|y\|_{[1-r, e+h]} \leq k\right\}$, we have $\|\mathfrak{S} y\|_{[1-r, e+h]} \leq \hat{L}$. For $y \in B$ and $s \in J$ we have

$$
\left\|y^{s}\right\|_{[-r, h]}=\max _{\theta \in[-r, h]}|y(s+\theta)| \leq \max _{t \in[1-r, e+h]}|y(t)|=\|y\|_{[1-r, e+h]}
$$

and

$$
\left\|y^{s}+u^{s}\right\| \leq\left\|y^{s}\right\|_{[-r, h]}+\left\|u^{s}\right\|_{[-r, h]} \leq\|y\|_{[-r, h]}+\max \left\{\|x\|_{[1-r, 1]},\|x\|_{[e, e+h]}\right\} .
$$

Let $y \in U_{k}$. Since $f$ is continuous, therefore, for $t \in[1, e]$, we have

$$
\begin{aligned}
|(\mathfrak{S} y)(t)| \leq & \mid \frac{1}{\Gamma(\alpha)} \int_{1}^{t}\left(\log \frac{t}{s}\right)^{\alpha-1} \frac{f\left(s, y^{s}+u^{s}\right)}{s} d s \\
& -(\log t)^{\alpha-1} \frac{1}{\Gamma(\alpha)} \int_{1}^{e}\left(\log \frac{e}{s}\right)^{\alpha-1} \frac{f\left(s, y^{s}+u^{s}\right)}{s} d s \mid
\end{aligned}
$$




$$
\begin{aligned}
\leq & \frac{1}{\Gamma(\alpha)} \int_{1}^{t}\left(\log \frac{t}{s}\right)^{\alpha-1} \frac{p(s) \Omega\left(\left\|y^{s}+u^{s}\right\|_{[-r, h]}\right)}{s} d s \\
& +(\log t)^{\alpha-1} \frac{1}{\Gamma(\alpha)} \int_{1}^{e}\left(\log \frac{e}{s}\right)^{\alpha-1} \frac{p(s) \Omega\left(\left\|y^{s}+u^{s}\right\|_{[-r, h]}\right)}{s} d s \\
\leq & \frac{2\|p\|_{0} \Omega\left(k+\max \left\{\|x\|_{[1-r, 1]},\|x\|_{[e, e+h]}\right\}\right)}{\Gamma(\alpha)} \int_{1}^{e}\left(\log \frac{t}{s}\right)^{\alpha-1} \frac{1}{s} d s \\
\leq & \frac{2\|p\|_{0} \Omega\left(k+\max \left\{\|x\|_{[1-r, 1]},\|x\|_{[e, e+h]}\right\}\right)}{\Gamma(\alpha+1)},
\end{aligned}
$$

and so

$$
\|\mathfrak{S} y\|_{[1-r, e+h]} \leq \frac{2\|p\|_{0} \Omega\left(k+\max \left\{\|x\|_{[1-r, 1]},\|x\|_{[e, e+h]}\right\}\right)}{\Gamma(\alpha+1)}:=\hat{L} .
$$

Consequently, $\mathfrak{S}$ maps bounded sets into bounded sets in $B$.

Step 3: $\mathfrak{S}$ maps bounded sets into equicontinuous sets of $B$.

Let $t_{1}, t_{2} \in[1, e]$ with $t_{1}<t_{2}$ and $U_{k}$ be a bounded set of $B$ as in Step 2. Let $y \in U_{k}$, then we have

$$
\begin{aligned}
& \left|(\mathfrak{S} y)\left(t_{2}\right)-(\mathfrak{S} y)\left(t_{1}\right)\right| \\
& \quad \leq \int_{1}^{e}\left|G\left(t_{2}, s\right)-G\left(t_{1}, s\right)\right| \frac{\left|f\left(s, y^{s}+u^{s}\right)\right|}{s} d s \\
& \quad \leq\|p\|_{0} \Omega\left(k+\max \left\{\|x\|_{[1-r, 1]},\|x\|_{[e, e+h]}\right\}\right) \int_{1}^{e}\left|G\left(t_{2}, s\right)-G\left(t_{1}, s\right)\right| \frac{d s}{s} .
\end{aligned}
$$

As $t_{1} \rightarrow t_{2}$ the right-hand side of the last inequality tends to zero. The equicontinuity for the cases $t_{1}<t_{2} \leq 0$ and $t_{1} \leq 0 \leq t_{2}$ is obvious.

In view of Steps 1 to 3 , it follows by the Arzelá-Ascoli theorem that the operator $\mathfrak{S}$ is continuous and completely continuous.

Step 4: A priori bounds.

We will show that there exists an open set $U \subset B$ with $y \neq \lambda \mathfrak{S} y$ for $0<\lambda<1$ and $y \in \partial U$. Let $y \in B$ and $y=\lambda \mathfrak{S} y$ for some $0<\lambda<1$. Thus, for each $t \in[1, e]$, we have

$$
y(t)=\lambda \int_{1}^{e} G(t, s) f\left(s, y^{s}+u^{s}\right) \frac{d s}{s} .
$$

This implies by our assumptions that, for each $t \in J$, we get

$$
\begin{aligned}
|y(t)| \leq & \frac{1}{\Gamma(\alpha)} \int_{1}^{t}\left(\log \frac{t}{s}\right)^{\alpha-1} \frac{p(s) \Omega\left(\left\|y^{s}+u^{s}\right\|_{[-r, h]}\right)}{s} d s \\
& +(\log t)^{\alpha-1} \frac{1}{\Gamma(\alpha)} \int_{1}^{e}\left(\log \frac{e}{s}\right)^{\alpha-1} \frac{p(s) \Omega\left(\left\|y^{s}+u^{s}\right\|_{[-r, h]}\right)}{s} d s \\
\leq & \frac{2\|p\|_{0} \Omega\left(\|y\|_{[-r, h]}+\max \left\{\|x\|_{[1-r, 1]},\|x\|_{[e, e+h]}\right\}\right)}{\Gamma(\alpha)} \int_{1}^{e}\left(\log \frac{e}{s}\right)^{\alpha-1} \frac{1}{s} d s \\
\leq & \frac{2\|p\|_{0}}{\Gamma(\alpha+1)} \Omega\left(\|y\|_{[-r, h]}+\max \left\{\|x\|_{[1-r, 1]},\|x\|_{[e, e+h]}\right\}\right) .
\end{aligned}
$$


Then

$$
\frac{\|y\|_{[1-r, e+h]}}{\frac{2\|p\|_{0}}{\Gamma(\alpha+1)} \Omega\left(\|y\|_{[-r, h]}+\max \left\{\|x\|_{[1-r, 1]},\|x\|_{[e, e+h]}\right\}\right)} \leq 1 .
$$

By $\left(H_{2}\right)$, there exists $K_{0}$ such that $\|y\|_{[1-r, e+h]} \neq K_{0}$. Set

$$
U=\left\{y \in B:\|y\|_{[1-r, e+h]}<K_{0}+1\right\} .
$$

By our choice of $U$, there is no $y \in \partial U$ such that $y=\lambda \mathfrak{S} y$ for some $0<\lambda<1$. As a consequence of the nonlinear alternative of Leray-Schauder type (Lemma 3.1), we deduce that $\mathfrak{S}$ has a fixed point $y \in \bar{U}$ which is a solution to problem (1)-(3). This completes the proof.

The next result, concerning the existence of a unique solution of problem (1)-(3), is based on the Banach fixed point theorem.

Theorem 3.3 Let $f:[1, e] \times C([-r, h], \mathbb{R}) \rightarrow \mathbb{R}$. Assume that there exists $L>0$ such that

$$
|f(t, u(t))-f(t, v(t))| \leq L\|u-v\|_{[-r, h]}
$$

for $t \in[1, e]$ and every $u, v \in C([-r, h], \mathbb{R})$.

If

$$
\frac{2 L}{\Gamma(\alpha+1)}<1
$$

then the BVP (1)-(3) has a unique solution on the interval $[1-r, e+h]$.

Proof As argued in the proof of the preceding theorem, it will be shown that the operator $\mathfrak{S}: B \rightarrow B$ defined by (13) is a contraction, where $B=\{y \in C([1-r, e+h], \mathbb{R}): y(1)=0\}$. For that, let $y_{1}, y_{2} \in B$. Then, for $t \in[1, e]$, we obtain

$$
\begin{aligned}
\left|\left(\mathfrak{S} y_{1}\right)(t)-\left(\mathfrak{S} y_{2}\right)(t)\right| & \leq \int_{1}^{e} G(t, s)\left|f\left(s, y_{1}^{s}+u^{s}\right)-f\left(s, y_{2}^{s}+u^{s}\right)\right| \frac{d s}{s} \\
& \leq L \int_{1}^{e} G(t, s)\left\|y_{1}^{s}-y_{2}^{s}\right\|_{[-r, h]} \frac{d s}{s} \\
& \leq \frac{2 L}{\Gamma(\alpha)}\left\|y_{1}-y_{2}\right\|_{[-r, h]} \int_{1}^{e}\left(\log \frac{e}{s}\right)^{\alpha-1} \frac{1}{s} d s \\
& \leq \frac{2 L}{\Gamma(\alpha+1)}\left\|y_{1}-y_{2}\right\|_{[1-r, e+h]} .
\end{aligned}
$$

Consequently, we get

$$
\left\|\mathfrak{S} y_{1}-\mathfrak{S} y_{2}\right\|_{[1-r, e+h]} \leq \frac{2 L}{\Gamma(\alpha+1)}\left\|y_{1}-y_{2}\right\|_{[1-r, e+h]},
$$

which implies that $\mathfrak{S}$ is a contraction by the given assumption, and hence $\mathfrak{S}$ has a unique fixed point by means of the Banach contraction mapping principle. This, in turn, implies that the problem (1)-(3) has a unique solution on the interval $[1-r, e+h]$. 


\section{Existence results for the problem (4)-(6)}

For the forthcoming analysis, we need the following lemmas.

Lemma 4.1 (Nonlinear alternative for Kakutani maps ([28])) Let E be a Banach space, $C$ a closed convex subset of $E, U$ an open subset of $C$ and $0 \in U$. Suppose that $F: \bar{U} \rightarrow \mathcal{P}_{c p, c}(C)$ is a upper semicontinuous compact map. Then either

(i) F has a fixed point in $\bar{U}$, or

(ii) there is $a u \in \partial U$ and $\lambda \in(0,1)$ with $u \in \lambda F(u)$.

Lemma 4.2 ([29]) Let $X$ be a Banach space. Let $F:[0,1] \times X \rightarrow \mathcal{P}_{c p, c}(X)$ be an $L^{1}$-Carathéodory multi-valued map and let $\Theta$ be a linear continuous mapping from $L^{1}([0,1], X)$ to $C([0,1], X)$. Then the operator

$$
\Theta \circ S_{F}: C([0,1], X) \rightarrow \mathcal{P}_{c p, c}(C([0,1], X)), \quad x \mapsto\left(\Theta \circ S_{F}\right)(x)=\Theta\left(S_{F, x}\right),
$$

is a closed graph operator in $C([0,1], X) \times C([0,1], X)$.

The next fixed point theorem is the well-known Covitz and Nadler fixed point theorem for multi-valued contractions [30] (see also Deimling, [24] Theorem 11.1).

Lemma 4.3 (Covitz and Nadler ([30])) Let $(X, d)$ be a complete metric space. If $G: X \rightarrow$ $\mathcal{P}_{c l}(X)$ is a contraction, then Fix $G \neq \emptyset$.

Theorem 4.4 Assume that $\left(H_{2}\right)$ holds. In addition, we suppose that the following conditions hold:

$\left(A_{1}\right) \quad F:[1, e] \times C([-r, h], \mathbb{R}) \rightarrow \mathcal{P}_{c p, c}(\mathbb{R})$ is an $L^{1}$-Carathéodory multi-valued map;

$\left(A_{2}\right)$ there exist $p \in C([1, e], \mathbb{R})$ and $\Omega:[0, \infty) \rightarrow(0, \infty)$ continuous and nondecreasing such that

$$
\|F(t, u)\|:=\sup \{|v|: v \in F(t, u)\} \leq p(t) \Omega\left(\|u\|_{[-r, h]}\right)
$$

for almost all $t \in[1, e]$ and all $u \in C([-r, h], \mathbb{R})$.

Then the problem (4)-(6) has at least one solution on the interval $[1-r, e+h]$.

Proof We transform the problem (4)-(6) into a fixed point problem. A solution to (4)-(6) is a fixed point of the operator $\mathcal{N}: C([1-r, e+h], \mathbb{R}) \longrightarrow \mathcal{P}(C([1-r, e+h], \mathbb{R}))$ defined by

$$
\mathcal{N}(x):= \begin{cases}h \in C([1-r, e+h], \mathbb{R}): & \\
h(t)=\left\{\begin{array}{ll}
\chi(t), & \text { if } t \in[1-r, 1], \\
\int_{0}^{t} G(t, s) v(s) \frac{d s}{s}, & \text { if } t \in[1, e], \\
\psi(t), & \text { if } t \in[e, e+h],
\end{array}\right\}\end{cases}
$$

where

$$
v \in S_{F, y}=\left\{v \in L^{1}([1, e], \mathbb{R}): v(t) \in F\left(t, y^{t}\right) \text { for a.e. } t \in J\right\} .
$$


As in the proof of Theorem 3.2, let $B=\{y \in C([1-r, e+h], \mathbb{R}): y(1)=0\}$ and let $\mathfrak{T}: B \rightarrow$ $\mathcal{P}(B)$ be defined by

$$
\mathfrak{T}(y):= \begin{cases}h \in C([1-r, E+h], \mathbb{R}): & \\
h(t)=\left\{\begin{array}{ll}
0, & \text { if } t \in[1-r, 1], \\
\int_{0}^{t} G(t, s) v(s) \frac{d s}{s}, & \text { if } t \in[1, e], \\
0, & \text { if } t \in[e, e+h] .
\end{array}\right\}\end{cases}
$$

Now we show that the operator $\mathfrak{T}$ has a fixed point which is equivalent to proving that the operator $\mathcal{N}$ has a fixed point. This, in turn, will imply that there exists a solution of the problem (4)-(6). We do it in several steps.

Claim $1 \mathfrak{T}(y)$ is convex for each $y \in C([1-r, e+h], \mathbb{R})$.

This claim is obvious, since $F$ has convex values.

Claim $2 \mathfrak{T}$ maps bounded sets into bounded sets in $C([1-r, e+h], \mathbb{R})$.

As is Step 2 of the proof of Theorem 3.2, let $U_{k}=\left\{y \in B:\|y\|_{[1-r, e+h]} \leq k\right\}$.

Let $y \in U_{k}$. Then, for each $h \in \mathfrak{T}(y)$, there exists $v \in S_{F, y}$ such that

$$
h(t)=\int_{1}^{e} G(t, s) v(s) \frac{d s}{s}, \quad t \in[1, e]
$$

and that

$$
\begin{aligned}
|h(t)| \leq & \left|\frac{1}{\Gamma(\alpha)} \int_{1}^{t}\left(\log \frac{t}{s}\right)^{\alpha-1} \frac{v(s)}{s} d s-(\log t)^{\alpha-1} \frac{1}{\Gamma(\alpha)} \int_{1}^{e}\left(\log \frac{e}{s}\right)^{\alpha-1} \frac{v(s)}{s} d s\right| \\
\leq & \frac{1}{\Gamma(\alpha)} \int_{1}^{t}\left(\log \frac{t}{s}\right)^{\alpha-1} \frac{p(s) \Omega\left(\left\|y^{s}+u^{s}\right\|_{[-r, h]}\right)}{s} d s \\
& +(\log t)^{\alpha-1} \frac{1}{\Gamma(\alpha)} \int_{1}^{e}\left(\log \frac{e}{s}\right)^{\alpha-1} \frac{p(s) \Omega\left(\left\|y^{s}+u^{s}\right\|_{[-r, h]}\right)}{s} d s \\
\leq & \frac{2\|p\|_{0} \Omega\left(k+\max \left\{\|x\|_{[1-r, 1]},\|x\|_{[e, e+h]}\right\}\right)}{\Gamma(\alpha+1)} .
\end{aligned}
$$

Thus

$$
\|h\|_{[1-r, e+h]} \leq \frac{2\|p\|_{0} \Omega\left(k+\max \left\{\|x\|_{[1-r, 1]},\|x\|_{[e, e+h]}\right\}\right)}{\Gamma(\alpha+1)}:=\hat{L} .
$$

This shows that $\mathfrak{T}$ maps bounded sets into bounded sets in $B$.

Claim $3 \mathfrak{T}$ maps bounded sets in $C([1-r, e+h], \mathbb{R})$ into equicontinuous sets.

We consider $B_{k}$ as in Claim 2 and let $h \in \mathfrak{T}(y)$ for $y \in B_{k}, k>0$. Now let $t_{1}, t_{2} \in[1, e]$ with $t_{2}>t_{1}$. Then we have

$$
\begin{aligned}
\left|h\left(t_{2}\right)-h\left(t_{1}\right)\right| & \leq \int_{1}^{e}\left|G\left(t_{2}, s\right)-G\left(t_{1}, s\right)\right|\left|f\left(s, y^{s}+u^{s}\right)\right| \frac{d s}{s} \\
& \leq\|p\|_{0} \Omega\left(k+\max \left\{\|x\|_{[1-r, 1]},\|x\|_{[e, e+h]}\right\}\right) \int_{1}^{e}\left|G\left(t_{2}, s\right)-G\left(t_{1}, s\right)\right| \frac{d s}{s} .
\end{aligned}
$$


Clearly the right-hand side of the last inequality tends to zero as $t_{1} \rightarrow t_{2}$. In view of Claims 2,3 , and the Arzelá-Ascoli theorem, we can conclude that $\mathfrak{T}: B \longrightarrow \mathcal{P}(B)$ is completely continuous.

In our next step, we show that $\mathfrak{T}$ is upper semicontinuous. It is well known [24], Proposition 1.2, that $\mathfrak{T}$ will be upper semicontinuous if we prove that it has a closed graph, since $\mathfrak{T}$ is already shown to be completely continuous.

Claim 4 T has closed graph.

Let $x_{n} \rightarrow x_{*}, h_{n} \in \mathfrak{T}\left(x_{n}\right)$, and $h_{n} \rightarrow h_{*}$. Then we need to show that $h_{*} \in \mathfrak{T}\left(x_{*}\right)$. Associated with $h_{n} \in \mathfrak{T}\left(x_{n}\right)$, there exists $v_{n} \in S_{F, x_{n}}$ such that, for each $t \in[0,1]$,

$$
h_{n}(t)=\frac{1}{\Gamma(\alpha)} \int_{1}^{t}\left(\log \frac{t}{s}\right)^{\alpha-1} \frac{v_{n}(s)}{s} d s-(\log t)^{\alpha-1} \frac{1}{\Gamma(\alpha)} \int_{1}^{e}\left(\log \frac{e}{s}\right)^{\alpha-1} \frac{v_{n}(s)}{s} d s
$$

Thus it suffices to show that there exists $v_{*} \in S_{F, x_{*}}$ such that, for each $t \in[0,1]$,

$$
h_{*}(t)=\frac{1}{\Gamma(\alpha)} \int_{1}^{t}\left(\log \frac{t}{s}\right)^{\alpha-1} \frac{\nu_{*}(s)}{s} d s-(\log t)^{\alpha-1} \frac{1}{\Gamma(\alpha)} \int_{1}^{e}\left(\log \frac{e}{s}\right)^{\alpha-1} \frac{v_{*}(s)}{s} d s
$$

Let us consider the linear operator $\Theta: L^{1}([0,1], \mathbb{R}) \rightarrow C([0,1], \mathbb{R})$ given by

$$
f \mapsto \Theta(v)(t)=\frac{1}{\Gamma(\alpha)} \int_{1}^{t}\left(\log \frac{t}{s}\right)^{\alpha-1} \frac{v(s)}{s} d s-(\log t)^{\alpha-1} \frac{1}{\Gamma(\alpha)} \int_{1}^{e}\left(\log \frac{e}{s}\right)^{\alpha-1} \frac{v(s)}{s} d s
$$

Observe that

$$
\begin{aligned}
\left\|h_{n}(t)-h_{*}(t)\right\|= & \| \frac{1}{\Gamma(\alpha)} \int_{1}^{t}\left(\log \frac{t}{s}\right)^{\alpha-1} \frac{\left(v_{n}(s)-v_{*}(s)\right)}{s} d s \\
& -(\log t)^{\alpha-1} \frac{1}{\Gamma(\alpha)} \int_{1}^{e}\left(\log \frac{e}{s}\right)^{\alpha-1} \frac{\left(v_{n}(s)-v_{*}(s)\right)}{s} d s \| \\
\rightarrow & 0, \quad \text { as } n \rightarrow \infty
\end{aligned}
$$

Thus, it follows by Lemma 4.2 that $\Theta \circ S_{F}$ is a closed graph operator. Further, we have $h_{n}(t) \in \Theta\left(S_{F, x_{n}}\right)$. Since $x_{n} \rightarrow x_{*}$, therefore, we have

$$
h_{*}(t)=\frac{1}{\Gamma(\alpha)} \int_{1}^{t}\left(\log \frac{t}{s}\right)^{\alpha-1} \frac{v_{*}(s)}{s} d s-(\log t)^{\alpha-1} \frac{1}{\Gamma(\alpha)} \int_{1}^{e}\left(\log \frac{e}{s}\right)^{\alpha-1} \frac{v_{*}(s)}{s} d s
$$

for some $v_{*} \in S_{F, x_{*}}$.

Claim 5 We will show that there exists an open set $U \subset B$ with $y \neq \lambda \mathfrak{T} y$ for $0<\lambda<1$ and $y \in \partial U$.

Let $y \in B$ be such that $y \in \lambda \mathfrak{T}(y)$ for some $0<\lambda<1$. Then there exists $v \in S_{F, y}$ such that

$$
y(t)=\lambda \int_{1}^{e} G(t, s) v(s) \frac{d s}{s}, \quad t \in[1, e]
$$


This implies by our assumptions that, for each $t \in[1, e]$, we have

$$
\begin{aligned}
|y(t)| \leq & \frac{1}{\Gamma(\alpha)} \int_{1}^{t}\left(\log \frac{t}{s}\right)^{\alpha-1} \frac{p(s) \Omega\left(\left\|y^{s}+u^{s}\right\|_{[-r, h]}\right)}{s} d s \\
& +(\log t)^{\alpha-1} \frac{1}{\Gamma(\alpha)} \int_{1}^{e}\left(\log \frac{e}{s}\right)^{\alpha-1} \frac{p(s) \Omega\left(\left\|y^{s}+u^{s}\right\|_{[-r, h]}\right)}{s} d s \\
\leq & \frac{2\|p\|_{0} \Omega\left(\|y\|_{[-r, h]}+\max \left\{\|x\|_{[1-r, 1]},\|x\|_{[e, e+h]}\right\}\right)}{\Gamma(\alpha)} \int_{1}^{e}\left(\log \frac{e}{s}\right)^{\alpha-1} \frac{1}{s} d s \\
\leq & \frac{2\|p\|_{0}}{\Gamma(\alpha+1)} \Omega\left(\|y\|_{[1-r, e+h]}+\max \left\{\|x\|_{[1-r, 1]},\|x\|_{[e, e+h]}\right\}\right) .
\end{aligned}
$$

Then

$$
\frac{\|y\|_{[1-r, e+h]}}{\frac{2\|p\|_{0}}{\Gamma(\alpha+1)} \Omega\left(\|y\|_{[1-r, e+h]}+\max \left\{\|x\|_{[1-r, 1]},\|x\|_{[e, e+h]}\right\}\right)} \leq 1 .
$$

By $\left(H_{2}\right)$, there exists $K_{0}$ such that $\|y\|_{[1-r, e+h]} \neq K_{0}$. Set

$$
U=\left\{y \in C([1-r, e+h], \mathbb{R}):\|y\|_{[1-r, e+h]}<K_{0}+1\right\} .
$$

From the choice of $U$ there is no $y \in \partial U$ such that $y \in \lambda \mathfrak{T}(y)$ for $\lambda \in(0,1)$. As a consequence of the Leray-Schauder alternative for Kakutani maps (Lemma 4.1), we deduce that $\mathfrak{T}$ has a fixed point, which implies that the problem (4)-(6) has at least one solution. This completes the proof.

Finally, we present an existence result for the problem (4)-(6) with nonconvex valued right-hand side by using Covitz and Nadler's fixed point theorem.

\section{Theorem 4.5 Suppose that:}

$\left(B_{1}\right) \quad F:[1, e] \times C([-r, h], \mathbb{R}) \longrightarrow \mathcal{P}_{c p}(\mathbb{R})$ has the property that $F(\cdot, y):[1, e] \longmapsto \mathcal{P}_{c p}(\mathbb{R})$ is measurable for each $y \in C([-r, h], \mathbb{R})$;

$\left(B_{2}\right)$ there exists $\ell \in C(J, \mathbb{R})$, such that

$$
H_{d}(F(t, u), F(t, \bar{u})) \leq \ell(t)\|u-\bar{u}\|_{[-r, h]} \quad \text { for every } u, \bar{u} \in C([-r, h], \mathbb{R}),
$$

and

$$
d(0, F(0, u)) \leq \ell(t), \quad \text { for a.e. } t \in[1, e]
$$

If

$$
\frac{2}{\Gamma(\alpha+1)}\|\ell\|_{0}<1
$$

then the problem (4)-(6) has at least one solution. 
Proof Transform the problem (4)-(6) into a fixed point problem by means of the multivalued operator $\mathfrak{T}: B \rightarrow \mathcal{P}(B)$ introduced in Theorem 4.4. We shall show that $\mathfrak{T}$ satisfies the assumptions of Lemma 4.3. The proof will be given in two steps.

Step 1: $\mathfrak{T}(y) \in \mathcal{P}_{c l}(B)$ for each $y \in B$.

Indeed, let $\left(y_{n}\right)_{n \geq 0} \in \mathfrak{T}(y)$ such that $y_{n} \longrightarrow \tilde{y}$ in $B$. Then $\tilde{y} \in B$ and there exists $g_{n} \in S_{F, y}$ such that, for each $t \in[1, e]$,

$$
y_{n}(t)=\int_{1}^{e} G(t, s) g_{n}(s) \frac{d s}{s} .
$$

Using $\left(B_{1}\right)$ together with the fact that $F$ has compact values, we may pass to a subsequence to see that $g_{n}$ converges weakly to $g$ in $L^{1}([0.1], \mathbb{R})$. Then $g \in S_{F, x}$ and, for each $t \in[1, e]$, we have

$$
y_{n}(t) \longrightarrow \tilde{y}(t)=\int_{1}^{e} G(t, s) g(s) \frac{d s}{s} .
$$

So $\tilde{y} \in \mathfrak{T}(y)$.

Step 2: There exists $\gamma<1\left(\gamma:=\frac{2}{\Gamma(\alpha+1)}\|\ell\|_{0}\right)$ such that

$$
H_{d}(\mathfrak{T}(y), \mathfrak{T}(\bar{y})) \leq \gamma\|y-\bar{y}\|_{[1-r, e+h]} \quad \text { for each } y, \bar{y} \in B .
$$

Let $y, \bar{y} \in B$ and $h \in \mathfrak{T}(y)$. Then there exists $g(t) \in F\left(t, y^{t}+u^{t}\right)$ such that

$$
h(t)=\int_{0}^{e} G(t, s) g(s) \frac{d s}{s},
$$

for each $t \in J$. From $\left(B_{2}\right)$ it follows that

$$
H_{d}\left(F\left(t, y^{t}+u^{t}\right), F\left(t, \bar{y}^{t}+\bar{u}^{t}\right)\right) \leq \ell(t)\|y-\bar{y}\|_{[-r, h]}, \quad t \in[1, e] .
$$

Hence there is $w \in F\left(t, \bar{y}^{t}+u^{t}\right)$ such that

$$
|g(t)-w| \leq \ell(t)\|y-\bar{y}\|_{[-r, h]}, \quad t \in[1, e] .
$$

Consider $U:[1, e] \rightarrow \mathcal{P}(E)$, given by

$$
U(t)=\left\{w \in E:|g(t)-w| \leq \ell(t)\|y-\bar{y}\|_{[-r, h]}\right\} .
$$

Since the multi-valued operator $V(t)=U(t) \cap F\left(t, \bar{y}^{t}+u^{t}\right)$ is measurable (see Proposition III.4 in [31]), there exists a function $\bar{g}(t)$, which is a measurable selection for $V$. So, $\bar{g}(t) \in F\left(t, \bar{y}^{t}+u^{t}\right)$ and

$$
|g(t)-\bar{g}(t)| \leq \ell(t)\|y-\bar{y}\|_{[-r, h]}, \quad \text { for each } t \in[1, e] .
$$

Let us define, for each $t \in[1, e]$,

$$
\bar{h}(t)=\int_{1}^{e} G(t, s) \bar{g}(s) \frac{d s}{s}
$$


and

$$
\begin{aligned}
|h(t)-\bar{h}(t)| & \leq \int_{1}^{e}|G(t, s)||g(s)-\bar{g}(s)| \frac{d s}{s} \\
& \leq \int_{1}^{e}|G(t, s)| \ell(s)\|y-\bar{y}\|_{[-r, h]} \frac{d s}{s} \\
& \leq \frac{2\|\ell\|_{0}\|y-\bar{y}\|_{[-r, h]}}{\Gamma(\alpha)} \int_{1}^{e}\left(\log \frac{e}{s}\right)^{\alpha-1} \frac{1}{s} d s \\
& \leq \frac{2}{\Gamma(\alpha+1)}\|\ell\|_{0}\|y-\bar{y}\|_{[-r, h]} .
\end{aligned}
$$

Thus

$$
\|h-\bar{h}\|_{[1-r, e+h]} \leq \frac{2}{\Gamma(\alpha+1)}\|\ell\|_{0}\|y-\bar{y}\|_{[1-r, e+h]} .
$$

Analogously, interchanging the roles of $y$ and $\bar{y}$, it follows that

$$
H_{d}(\mathfrak{T}(y), \mathfrak{T}(\bar{y})) \leq \frac{2}{\Gamma(\alpha+1)}\|\ell\|_{0}\|y-\bar{y}\|_{[1-r, e+h]} .
$$

So, $\mathfrak{T}$ is a contraction and hence, by Lemma $4.3, \mathfrak{T}$ has a fixed point $y$, which is a solution to $(4)-(6)$.

\section{Conclusions}

We have obtained several existence results for boundary value problems of Hadamardtype fractional differential equations and inclusions involving both retarded and advanced arguments by applying some standard tools of fixed point theory for single-valued and multi-valued maps. Our results are new and yield several new ones by fixing the parameters $r$ and $h$ appropriately. For example, the results for ordinary Hadamard-type fractional differential equations/inclusions follow by taking $r=h=0$. Our results reduce to the retarded and advanced argument cases for $r>0, h=0$ and $r=0, h>0$, respectively. The mixed (both retarded and advanced) case follows by choosing $r>0$ and $h>0$.

Competing interests

The authors declare that they have no competing interests.

Authors' contributions

Each of the authors, RPA, SKN, BA, and AKA contributed to each part of this work equally and read and approved the final version of the manuscript.

\section{Author details}

'Department of Mathematics, Texas A\&M University, Kingsville, TX 78363-8202, USA. ²Department of Mathematics, University of loannina, loannina, 451 10, Greece. ${ }^{3}$ Nonlinear Analysis and Applied Mathematics (NAAM)-Research Group, Department of Mathematics, Faculty of Science, King Abdulaziz University, P.O. Box 80203, Jeddah, 21589, Saudi Arabia.

\section{Acknowledgements}

This project was funded by the Deanship of Scientific Research (DSR) at King Abdulaziz University, under grant no. $\mathrm{HiCi}-46-130-36$. The authors, therefore, acknowledge with thanks DSR for technical and financial support. The authors also acknowledge the referees for their constructive remarks. 
References

1. Agrawal, OP: Analytical schemes for a new class of fractional differential equations. J. Phys. A 40(21), 5469-5477 (2007)

2. Ahmad, B, Ntouyas, SK: A fully Hadamard type integral boundary value problem of a coupled system of fractional differential equations. Fract. Calc. Appl. Anal. 17, 348-360 (2014)

3. Darwish, MA, Ntouyas, SK: Existence results for a fractional functional differential equation of mixed type. Commun. Appl. Nonlinear Anal. 15, 47-55 (2008)

4. El-Sayed, AMA, Gaafar, FM: Fractional calculus and some intermediate physical processes. Appl. Math. Comput. 144(1), 117-126 (2003)

5. Graef, JR, Kong, L, Wang, M: Existence and uniqueness of solutions for a fractional boundary value problem on a graph. Fract. Calc. Appl. Anal. 17, 499-510 (2014)

6. Hilfer, R: Applications of Fractional Calculus in Physics. World Scientific, Singapore (2000)

7. Kilbas, AA, Srivastava, HM, Trujillo, Jj: Theory and Applications of Fractional Differential Equations. North-Holland Mathematics Studies, vol. 204. Elsevier, Amsterdam (2006)

8. Kirane, M, Ahmad, B, Alsaedi, A, Al-Yami, M: Non-existence of global solutions to a system of fractional diffusion equations. Acta Appl. Math. 133, 235-248 (2014)

9. Miller, KS, Ross, B: An Introduction to the Fractional Calculus and Differential Equations. Wiley, New York (1993)

10. Podlubny, I: Fractional Differential Equations. Academic Press, San Diego (1999)

11. Punzo, F, Terrone, G: On the Cauchy problem for a general fractional porous medium equation with variable density Nonlinear Anal. 98, 27-47 (2014)

12. Samko, SG, Kilbas, AA, Marichev, OI: Fractional Integrals and Derivatives: Theory and Applications. Gordon \& Breach, Amsterdam (1993) (Russian edition, 1987)

13. Saxena, RK, Kalla, SL: On a fractional generalization of free electron laser equation. Appl. Math. Comput. 143, 89-97 (2003)

14. Saxena, RK, Mathai, AM, Haubold, HL: On generalized fractional kinetic equations. Physica A 344, 657-664 (2004)

15. Baleanu, D, Rezapour, S, Salehi, S: On the existence of solutions for a fractional finite difference inclusion via three points boundary conditions. Adv. Differ. Equ. 2015, 242 (2015)

16. Agarwal, RP, Baleanu, D, Hedayati, V, Rezapour, S: Two fractional derivative inclusion problems via integral boundary condition. Appl. Math. Comput. 257, 205-212 (2015)

17. Gambo, Y, Jarad, F, Baleanu, D, Abdeljawad, T: On Caputo modification of the Hadamard fractional derivatives. Adv. Differ. Equ. 2014, 10 (2014)

18. Yang, X-J, Srivastava, HM: An asymptotic perturbation solution for a linear oscillator of free damped vibrations in fractal medium described by local fractional derivatives. Commun. Nonlinear Sci. Numer. Simul. 29, 499-504 (2015)

19. Yu, C, Gao, G: Existence of fractional differential equations. J. Math. Anal. Appl. 310, 26-29 (2005)

20. Zhao, $X$, Yang, $H T$, He, YQ: Identification of constitutive parameters for fractional viscoelasticity. Commun. Nonlinear Sci. Numer. Simul. 19, 311-322 (2014)

21. Kolmanovskii, V, Myshkis, A: Introduction to the Theory and Applications of Functional-Differential Equations. Mathematics and Its Applications, vol. 463. Kluwer Academic, Dordrecht (1999)

22. Hale, J, Verduyn Lunel, SM: Introduction to Functional Differential Equations. Applied Mathematical Sciences, vol. 99. Springer, New York (1993)

23. Ahmad, B, Ntouyas, SK: Initial value problems of fractional order Hadamard-type functional differential equations. Electron. J. Differ. Equ. 2015, 77 (2015)

24. Deimling, K: Multivalued Differential Equations. de Gruyter, Berlin (1992)

25. Gorniewicz, L: Topological Fixed Point Theory of Multivalued Mappings. Mathematics and Its Applications, vol. 495. Kluwer Academic, Dordrecht (1999)

26. Hu, S, Papageorgiou, N: Handbook of Multivalued Analysis, Theory I. Kluwer Academic, Dordrecht (1997)

27. Tolstonogov, AA: Differential Inclusions in a Banach Space. Kluwer Academic, Dordrecht (2000)

28. Granas, A, Dugundji, J: Fixed Point Theory. Springer, New York (2005)

29. Lasota, A, Opial, Z: An application of the Kakutani-Ky Fan theorem in the theory of ordinary differential equations Bull. Acad. Pol. Sci., Sér. Sci. Math. Astron. Phys. 13, 781-786 (1965)

30. Covitz, H, Nadler, SB Jr:: Multivalued contraction mappings in generalized metric spaces. Isr. J. Math. 8, 5-11 (1970)

31. Castaing, C, Valadier, M: Convex Analysis and Measurable Multifunctions. Lecture Notes in Mathematics, vol. 580. Springer, Berlin (1977)

\section{Submit your manuscript to a SpringerOpen ${ }^{\circ}$ journal and benefit from:}

- Convenient online submission

- Rigorous peer review

Immediate publication on acceptance

- Open access: articles freely available online

- High visibility within the field

- Retaining the copyright to your article 